\title{
Low-pressure gas chromatography - ion trap mass spectrometry for the fast determination of polycyclic aromatic hydrocarbons in air samples
}

\author{
Khaiwal Ravindra*, Ana F.L. Godoi ${ }^{1}$, László Bencs ${ }^{2}$, René Van Grieken
}

\section{Micro and Trace Analysis Center, Department of Chemistry, University of Antwerp, Universiteitsplein 1, B-2610 Antwerp, Belgium}

\section{Abstract}

The low-pressure gas chromatography - ion trap mass spectrometry (LPGC-ITMS) method was investigated to shorten the analysis time for 18 US Environmental Protection Agency priority listed polycyclic aromatic hydrocarbons (PAHs). Their elution was optimised with a short, wide-bore column coupled to a deactivated capillary at the inlet end and with a long, conventional column to compare their analytical performance. The analytical figures of merit under optimal LPGC-ITMS conditions were determined with respect to chromatographic separation, $\mathrm{S} / \mathrm{N}$ ratio, limit of detection and precision. The peak width at half height of $1.5 \mathrm{~s}$ matched the duty cycle of the ITMS. Up to 16 PAHs in the molecular weight (MW) range of 128-278 Da could be separated in a very short time, i.e. less than 13 min using LPGC-ITMS, whereas with conventional GC-MS, it took approximately $40 \mathrm{~min}$. However, LPGC-ITMS has a limited loss of separation power compared to that of conventional GC-MS due to occurrence of 3 critical pairs for high MW PAHs. For a practical evaluation, the LPGC-ITMS approach was applied to the determination of PAHs in gas and aerosol phase samples collected in the ambient air of Hasselt, Belgium.

Keywords: PAHs analysis, fast GC method, LPGC-ITMS, environmental monitoring.

* Corresponding author, Khaiwal Ravindra, Khaiwal.Ravindra@ua.ac.be or Khaiwal@yahoo.com, Tel.: +32 3820 2384; Fax: +32 38202376

Permanent addresses: ${ }^{1}$ Centro Universitário Positivo (UnicenP), Curitiba - PR, Brazil; ${ }^{2}$ Research Institute for Solid State Physics and Optics, Hungarian Academy of Sciences, P.O. Box 49, H-1525 Budapest, Hungary 


\section{Introduction}

The application of vacuum column-outlet conditions in a short, wide-bore column is an attractive way to increase the speed of gas chromatography (GC) analysis, whereas its compatibility with an ion trap (IT) or a quadrupole mass spectrometer (MS) remains retained [1-4]. Despite the attractive speed and larger loadability offered by a wide-bore column operated under vacuum outlet conditions or by low-pressure gas chromatography ion trap mass spectrometry (LPGC-ITMS), i.e., when a GC is used with IT detector; there are only few applications of this technique available [5-8]. Polycyclic aromatic hydrocarbons (PAHs) are ubiquitous and of major health concern, mainly due to their wellknow carcinogenic and mutagenic properties [9]. Therefore, in the present study, an application of LPGC-ITMS was elaborated and applied to a very fast determination of United States Environmental Protection Agency (US EPA) priority listed 18 PAHs [10] in air samples. The analytical performance of the LPGC-ITMS method was compared to that of a common GC-MS method with a conventional column. Further, LPGC-ITMS was applied to the determination of PAHs in ambient aerosol and gas phase samples.

\section{Experimental}

\section{Instrumentation}

A Varian Saturn 2000 IT-MS system was used in combination with a Varian 3800 gas chromatograph (Walnut Creek, CA, USA), equipped with a Varian 1079 universal injector, being used in splitless mode. Samples were injected with a Varian 8200 autosampler. For the conventional method, a non-polar CP-Sil 8 column (30 m x $0.32 \mathrm{~mm}$ internal diameter (I.D.); film thinkness $\left(\mathrm{d}_{\mathrm{f}}\right)=1 \mu \mathrm{m}$, Varian Chrompack, Middelburg, The Netherlands) was applied. For fast GC analysis, a shorter, but wider CP-Sil 8 column (10 m x $0.53 \mathrm{~mm}$ I.D.; $\mathrm{d}_{\mathrm{f}}=1 \mu \mathrm{m}$, Varian Chrompack, Middelburg, The Netherlands) was used. The column was coupled to an uncoated restriction column of $60 \mathrm{~cm}$ x $0.1 \mathrm{~mm}$ I.D. (Varian Chrompack) by a single ferrule column connector. Helium (Air Liquide, Liege, Belgium) was used as a carrier gas for both methods.

\section{Reagents and standards}

The 18 US EPA priority listed PAHs were used either separately or in a mixture of standard solutions for calibration. These PAHs include naphthalene, acenaphthene, 
acenapthylene, fluorene, phenanthrene, anthracene, fluoranthene, pyrene, benzo[a]anthracene, chrysene, benzo[b]fluoranthene, benzo[k]fluoranthene, benzo[e]pyrene, benzo[a]pyrene, perylene, indeno[1,2,3-cd]pyrene, dibenz[a,h]anthracene, benzo[ghi]perylene. Further, a mixture of five perdeuterated PAHs $\left(\left[{ }^{2} \mathrm{H}_{12}\right]\right.$ perylene, $\left[{ }^{2} \mathrm{H}_{12}\right]$ chrysene, $\left[{ }^{2} \mathrm{H}_{8}\right]$ naphthalene, $\left[{ }^{2} \mathrm{H}_{10}\right]$ phenanthrene, and $\left[{ }^{2} \mathrm{H}_{8}\right]$ acenaphthene) was used as internal standards. All chemicals were of analytical reagent grade.

\section{Samples preparation}

Air samples were collected with a high volume sampler (Anderson, OH, USA) from the ambient air in Hasselt, Belgium, during October-November 2002, as a part of an environmental monitoring programme of the Flemish Environment Agency (VMM). The details about the sampling [11] and extraction procedures are described in detail elsewhere $[11,12]$. The extracts were concentrated under a gentle flow of $\mathrm{N}_{2}$ up to dryness and were re-dissolved in $50 \mu \mathrm{L}$ isooctane. Recovery efficiencies for $18 \mathrm{PAHs}$ were found to be between 80 and $120 \%$ with the certified reference material of the National Institute of Standards and Technology (NIST): SRM1650a (Diesel Particulate Matter). The NIST standard PAHs mixture (SRM 1647d, Schmidt, Amsterdam, The Netherlands) is used for the calibration of analytical methods and for spiking the samples.

\section{Results and discussion}

\section{Optimization of LPGC-ITMS conditions}

Table 1 lists various GC parameters such as temperature programming rate, injection temperature, flow rate, and injected volume that were studied to achieve optimal separation of PAHs with low and high molecular weight (MW). The analyses were performed by selected ion monitoring (SIM), i.e., measuring the molecular ion of each compound. Standard mixtures were injected in split and splitless mode. The latter operation provided increased sensitivity. The injected amounts of individual PAHs between 2.5 and $15 \mathrm{ng}$ by the use of $0.5-3 \mu \mathrm{L}$ volume from a standard solution with a concentration of 5 $\mathrm{ng} / \mu \mathrm{L}$ had no significant effect on the separation efficiency. The fast temperature programming $\left(40{ }^{\circ} \mathrm{C} / \mathrm{min}\right)$ starting from an initial temperature of $40{ }^{\circ} \mathrm{C}$ onwards gave the best combination of adequate separation of PAHs and reduction of the analysis time. Specifically, baseline separation was obtained for the PAHs with MW of 128, 152, 154, 166, 178 and 202 Da. 
In comparison with GC-MS using a conventional column, most of the mass spectral parameters such as scan rate, emission current, maximum ionisation time and multiplier offset did not require major changes for LPGC-ITMS (Table 1). The scanrate was increased to $3 \mathrm{scan} / \mathrm{s}$ to improve the resolution of the chromatographic peak profiles.

\section{Comparison of conventional GC-MS and LPGC-ITMS for the determination of PAHS}

The analysis of a standard PAH mixture was carried out using conventional GC-MS and LPGC-ITMS methods under optimized conditions. Fig. 1 shows the corresponding total ion current chromatograms. The elution on the conventional column was optimised following the US EPA Method TO-13A [10], resulting in a total analysis time of $40 \mathrm{~min}$. In contrast, the use of LPGC-ITMS allowed the elution time to be reduced to less than $13 \mathrm{~min}$. A further advantage of the method was the low elution temperature that in turn, significantly reduced the background, due to column bleeding. For a detailed evaluation of the chromatographic separation obtained on conventional and LPGC-ITMS columns, the mass chromatograms of individual PAHs were compared. In general, the peak width at half height $\left(W_{h}\right)$ was found to be narrower with the LPGC-ITMS than with the conventional method, e.g., yielding for fluoranthene the values of 1.2 and $2.1 \mathrm{~s}$, respectively. Furthermore, the LPGC-ITMS method fully retained the chromatographic separation efficiency of GC-MS with a conventional column for PAHs in the MW range of 128-202 Da. However, the reduction in the analysis time sacrificed some resolution for PAH analogs with MW between 228 and 252 Da, i.e. occurrence of 3 critical pairs of PAHs, two of them separated below the half height. Since the quantitation was performed in SIM with other target ions, it was possible to identify and quantify them based on their separation near half height and the slight difference in their retention times. Therefore, we were able to determine 16 PAHs except for benzo[b]fluoranthene and benzo[k]fluoranthene, which were quantified in total. Interestingly, LPGC-ITMS allows high MW PAHs (276, $278 \mathrm{Da})$ to be eluted with adequate separation, but much faster than in the case of a conventional column (peaks 16, 17 on Fig. 1). The calibration curves based on diluted standards showed correlation coefficients better than 0.999 for each PAH. Table 2 lists the separation power of the two methods expressed as the numbers of theoretical plates $(n)$ by the formula of $n=$ $5.545\left(t_{r} / W_{h}\right)^{2}$, where $t_{r}$ is the retention time of the peak.

Analysis of serial dilutions of PAH standards shows that the absolute limits of detection (LOD) for the MW range from 128 to $202 \mathrm{Da}$ varied from $50 \mathrm{pg}$ to $140 \mathrm{pg}$, and 
are comparable to those of the conventional method (65 to $120 \mathrm{pg}$ ). Unfortunately, this observation does not extend to the PAH analogues between 228-252 Da, because the loss in chromatographic separation comes together with a significant reduction in the detection capabilities. The LOD in this range varied from 70 to $150 \mathrm{pg}$, except for benzo[a]anthracene $(650 \mathrm{pg})$ and perylene (1000 pg). In contrast, the high MW PAHs (276 and $278 \mathrm{Da}$ ) were detected with proper resolution and with a similar sensitivity to that of the conventional method. A LOD of $320 \mathrm{pg}$ was reported for indeno[1,2,3-cd]pyrene and benzo[ghi]perylene, while it was $1650 \mathrm{pg}$ for dibenz[a,h]anthracene. The comparative LOD values in conventional columns lies in the range of $750 \mathrm{pg}$ on column. The above LOD data are also comparable with those reported by Sheu et al. [13] and Sofuoglu et al. [14]. However, it has to be noted that the larger loadability of a wide-bore capillary column may further improve the LOD.

Additional parameters for a trace analysis method, the precision and linear range of quantitation were observed to be adequate for quantitation of PAHs. Repeated (18) injections of a PAHs mixture with a concentration of $2.5 \mathrm{ng} \mathrm{\mu L}^{-1}$ shows relative standard deviations of 5-15\%. This analytical performance of LPGC-ITMS is considered to be adequate for a fast routine monitoring of PAH levels in environmental samples.

\section{Practical evaluation of the LPGC-ITMS}

The performance of the LPGC-ITMS method was evaluated by the analysis of air samples collected from Hasselt (Belgium) near a highway. During the monitoring period the daily average concentrations of total PAHs $\left(\sum 18\right)$ varied from $24 \pm 4 \mathrm{ng} \mathrm{m}^{-3}$ to $33 \pm 4 \mathrm{ng} \mathrm{m}^{-3}$ in gas phase samples, whereas it ranged from $6.6 \pm 1.3 \mathrm{ng} \mathrm{m}^{-3}$ to $8.5 \pm 0.6 \mathrm{ng} \mathrm{m}^{-3}$ for the aerosol. The daily levels of individual PAHs in the gas phase samples varied from below the LOD to $19 \mathrm{ng} \mathrm{m}^{-3}$, and showed the prevalence of low and medium MW PAHs, such as phenanthrene, $16 \pm 3.1 \mathrm{ng} \mathrm{m}^{-3}$; fluorene, $3.3 \pm 0.7 \mathrm{ng} \mathrm{m}^{-3}$; fluoranthene, $2.4 \pm 1.0 \mathrm{ng} \mathrm{m}^{-3}$; and pyrene, $2.0 \pm 1.0 \mathrm{ng} \mathrm{m}^{-3}$. The particulate phase reflected the occurrence of predominantly high MW PAHs (dibenz[a,h]anthracene, $2.5 \pm 1.9 \mathrm{ng} \mathrm{m}^{-3}$; benzo[ghi]perylene, $2.2 \pm 1.5 \mathrm{ng} \mathrm{m}^{-}$ 3; and indeno[1,2,3-cd]pyrene, $1.9 \pm 1.0 \mathrm{ng} \mathrm{m}^{-3}$ ). The daily individual PAH concentrations ranged up to $3.9 \mathrm{ng} \mathrm{m}^{-3}$, whereas the more volatile PAHs were found to be at lower levels in the particulate matter.

\section{Conclusion}


Compared to the conventional GC-MS methodology, LPGC-ITMS provides a valuable alternative for a fast analysis of PAHs in air samples. This new method allows the analysis time to be reduced by a factor of three with the preservation of the chromatographic resolution for the low MW PAHs that are prevalent in the gas phase samples of the ambient air. The loss of separation power for high MW PAHs is an acceptable shortcoming, when the increased sample throughput is taken into account and when information on concentration of all individual (e.g. 18 US EPA) PAHs is not required. Furthermore, LPGC-ITMS is an affordable method that can be readily implemented on current GC-MS systems without any major change in the instrumental configuration.

\section{Acknowledgements}

The authors thank to Prof. Luc Van Vaeck and Dr. Peter Joos (University of Antwerp), and Dr. Eric Wauters (VMM), for their help and suggestions. This work was partially supported by the EU within research project EVK4 CT 2001-00067. 


\section{References}

[1] P.A. LeClercq, G.J. Scherpenzeel, E.A.A. Vermeer, J. Chromatogr. 241 (1982) 61.

[2] P.A. LeClercq, C.A. Cramers, Mass Spectrom. Rev. 17 (1998) 37.

[3] M.V. Deursen, H.G. Janssen, J. Beens, P. Lipman, R. Reinierkens, G. Rutten, C.A. Cramers, J. Microcol. Sep. 12 (2000) 613.

[4] J. de Zeeuw, J. Peene, H.G. Janssen, X. Lou, J. High Resolut. Chromatogr. 23 (2000) 677.

[5] K. Mastovska, S.J. Lehotay, J. Hajslova, J. Chromatogr. A 926 (2001) 291.

[6] P.E. Joos, A.F.L. Godoi, R. De Jong, J. De Zeeuw, R. Van Grieken, J. Chromatogr. A, 985 (2004) 191.

[7] A.F.L. Godoi, W. Vilegas, R.H.M. Godoi, L. Van Vaeck, R. Van Grieken, J. Chromatogr. A 1027 (2004) 127.

[8] A.F.L. Godoi, K. Ravindra, L. Van Vaeck, R. Van Grieken, J. Chromatogr. A 1027 (2004) 49.

[9] K. Ravindra, A.K. Mittal, R. Van Grieken, Rev. Environ. Health 16 (2001) 169.

[10] Compendium Method TO-13A, Second adition, EPA/625/R-96/010b, January 1999, EPA, Cincinnati, USA.

[11] K. Ravindra, L. Bencs, E. Wauters, J. de Hoog, F. Deutsch, E. Roekens, N. Bleux, P. Berghmans, R. Van Grieken, Atmos. Environ. 40 (2006) 771.

[12] K. Ravindra, E. Wauters, S.K. Taygi, S. Mor, R. Van Grieken, Environ. Monit. Assess. (2006), PIPS No: DO00017051.

[13] H.L. Sheu, W.J. Lee, S.J. Lin, G.C. Fang, H.C. Chang, W.C. You, Environ. Pollut. 96 (1997) 369.

[14] A. Sofuoglu, M. Odabasi, Y. Tasdemir, N.R. Khalili, T.M. Holsen, Atmos. Environ. 35 (2001) 6503. 
Table 1. Chromatographic and mass spectrometric parameters studied for the optimization of PAH analysis using the LPGC-ITMS and conventional GC-MS

\begin{tabular}{lccc}
\hline Parameter & Studied range & \multicolumn{2}{c}{ Optimised values } \\
\cline { 3 - 4 } & & LP-GC & Conventional \\
\hline Temperature programming rate $\left({ }^{\circ} \mathrm{C}\right.$ min $\left.^{-1}\right)$ & $0-60$ & $a$ & $b$ \\
Injector temperature $\left({ }^{\circ} \mathrm{C}\right)$ & $200-300$ & 290 & 290 \\
Gas flow rate $\left(\mathrm{ml} \mathrm{min}^{-1}\right)$ & $1-5$ & 1.2 & 2.0 \\
Injection volume $(\mu \mathrm{l})$ & $0.5-10$ & 1 & 1 \\
Transfer line temperature $\left({ }^{\circ} \mathrm{C}\right)$ & $200-370$ & 230 & 240 \\
Ion trap temperature $\left({ }^{\circ} \mathrm{C}\right)$ & $200-260$ & 220 & 230 \\
Scan time $\left(\mathrm{s} \mathrm{scan}{ }^{-1}\right)$ & $0.22-0.8$ & 0.35 & 0.50 \\
Multiplier offset $(\mathrm{V})$ & -20 to +50 & 0 & 0 \\
Emission current $(\mu \mathrm{A})$ & $20-60$ & 50 & 50 \\
Max. ionization time $(\mathrm{ms})$ & $10-50$ & 30 & 35 \\
\hline
\end{tabular}

$10-50$

30

${ }^{\mathrm{a}}\left[40^{\circ} \mathrm{C}(1 \mathrm{~min}) \rightarrow 120^{\circ} \mathrm{C}\left(40^{\circ} \mathrm{C} \mathrm{m^{-1 }}\right) \rightarrow 260^{\circ} \mathrm{C}\left(15^{\circ} \mathrm{C} \mathrm{m^{-1 }}\right)\right]$

Table 2. Comparison of theoretical plate numbers calculated for PAH analysis with conventional GC-MS and LPGC-ITMS methods

\begin{tabular}{|l|c|c|}
\hline Compounds & \multicolumn{2}{|c|}{$\begin{array}{c}\text { Theoretical Plate Numbers* } \\
\text { LPGC-ITMS }\end{array}$} \\
\hline Naphthalene & 1711 & 40 \\
\hline Acenapthylene & 1853 & 143 \\
\hline Phenanthrene & 1277 & 204 \\
\hline Anthracene & 2461 & 456 \\
\hline Fluoranthene & 1790 & 486 \\
\hline Pyrene & 1800 & 565 \\
\hline Benzo[e]pyrene & 930 & 646 \\
\hline Benzo[a]pyrene & 884 & 563 \\
\hline Dibenz[a,h]anthracene & 360 & 466 \\
\hline
\end{tabular}



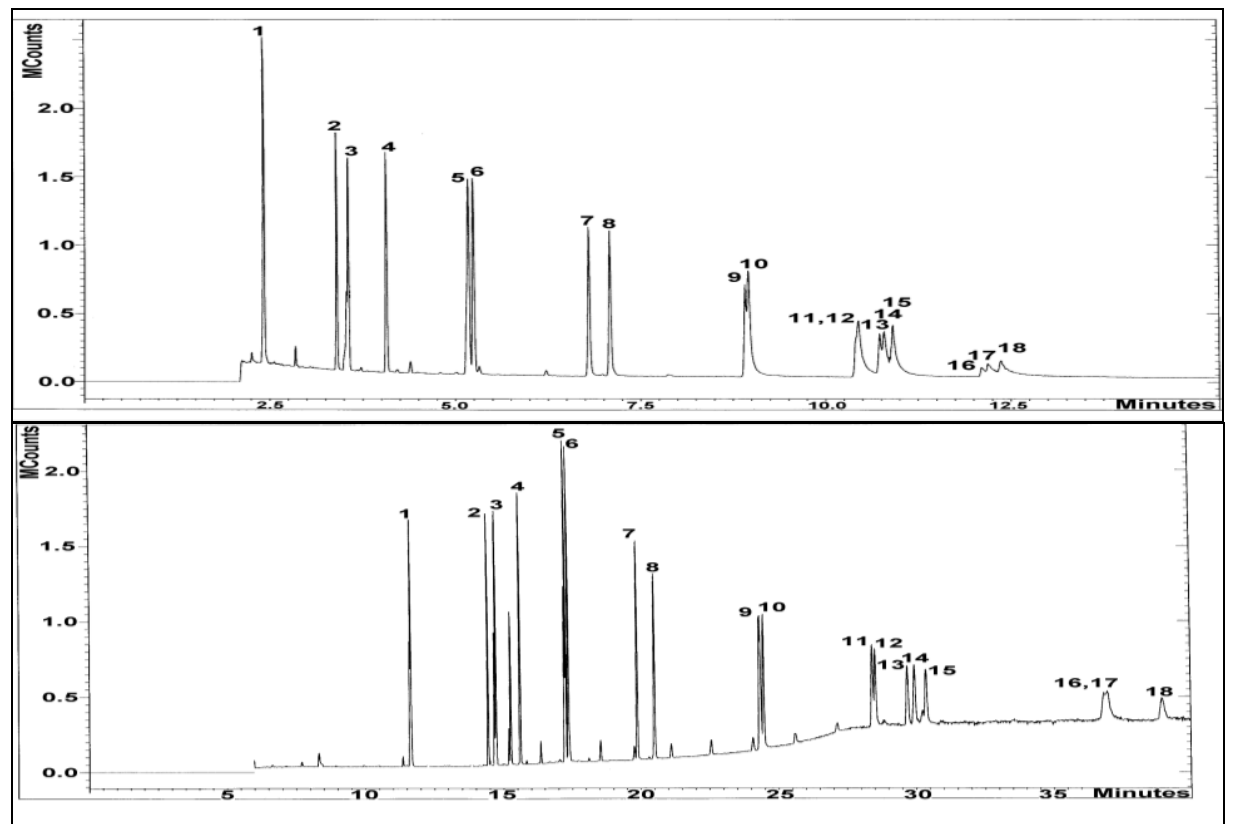

Figure 1. 


\section{Figure caption}

Figure 1. Comparison of the analysis of a PAH mixture using LPGC-ITMS (top) and CPSil 8 conventional column (bottom). Naphthalene (1), acenaphthene (2), acenapthylene (3), fluorene (4), phenanthrene (5), anthracene (6), fluoranthene (7), pyrene (8), benzo[a]anthracene (9), chrysene (10), benzo[b]fluoranthene (11), benzo[k]fluoranthene (12), benzo[e]pyrene (13), benzo[a]pyrene (14), perylene (15), indeno[1,2,3-cd]pyrene (16), dibenz[a,h]anthracene (17), benzo[ghi]perylene (18). 